\section{Istituto}

Nazionale

Fisica

Nucleare
Sezione SANITÀ

Istituto Superiore di Sanità

Viale Regina Elena 299

I-00161 Roma, Italy

INFN-ISS $97 / 10$

September 1997

\title{
HEAVY-QUARK BINDING AND KINETIC ENERGIES IN HEAVY-LIGHT MESONS AND THE CONSTITUENT QUARK MODEL
}

\author{
Silvano Simula \\ Istituto Nazionale di Fisica Nucleare, Sezione Sanità, \\ Viale Regina Elena 299, I-00161 Roma, Italy
}

\begin{abstract}
The spin-averaged binding energy and the hyperfine mass splitting of heavy-light mesons are investigated within the constituent quark model as a function of the inverse heavy-quark mass. It is shown that the so-called heavy-quark kinetic energy, $-\lambda_{1} / 2 m_{Q}$, may differ remarkably from the non-relativistic expectation $\left\langle p^{2}\right\rangle / 2 m_{Q}$, thanks to relativistic effects in the effective interquark potential for heavy-light mesons, which may yield substantial $1 / m_{Q}$ corrections to the heavy-quark static limit. The determination of the difference of the hadronic parameter $\lambda_{1}$ in the $B_{u(d)}$ and $B_{c}$ mesons can provide information about the strength of relativistic effects in the interquark interaction.
\end{abstract}

PACS numbers: 12.39.Ki; 12.39.Pn; 12.39. Hg

\footnotetext{
${ }^{a}$ To appear in Physics Letters B.
} 
The Heavy Quark Effective Theory (HQET) [1] is widely recognized as a powerful tool to investigate the properties of hadrons containing a heavy quark. This theory is based on an effective Lagrangian, written in terms of effective fields, which in the limit of infinite heavy-quark masses $\left(\mu_{Q} \rightarrow \infty\right)$ exhibits a spin-flavour symmetry, the so-called Heavy Quark Symmetry $(H Q S)$. Such a symmetry is shared by, but not manifest in $Q C D$ and it is only softly broken by terms of order $\Lambda_{Q C D} / \mu_{Q}$ [2], where $\Lambda_{Q C D}$ is the $Q C D$ scale parameter. The $H Q S$ allows to derive several exact relations among hadronic properties in the heavyquark limit, while at finite values of $\mu_{Q}$ the $H Q E T$ allows to represent physical quantities as a systematic expansion in terms of powers of the inverse heavy-quark mass, $1 / \mu_{Q}$, i.e. it organizes the structure of the power corrections to the HQS limit. However, neither the $H Q S$ nor the HQET can help to predict all the properties of heavy hadrons, because the calculation of the matrix elements of the relevant operators requires the knowledge of the non-perturbative structure of heavy hadrons. In this letter, we will focus on some of the relevant HQET matrix elements, particularly the hadronic parameters $\bar{\Lambda}, \lambda_{1}$ and $\lambda_{2}$, defined as

$$
\begin{aligned}
\bar{\Lambda} & =\lim _{\mu_{Q} \rightarrow \infty}\left[M^{(j)}\left(\mu_{Q}\right)-\mu_{Q}\right] \\
\lambda_{1} & =\left\langle M_{\infty}\left|\bar{h}(v)\left(i D_{\perp}\right)^{2} h(v)\right| M_{\infty}\right\rangle \\
\lambda_{2} & =\frac{g}{2 d_{j}}\left\langle M_{\infty}\left|\bar{h}(v) \sigma_{\mu \nu} G^{\mu \nu} h(v)\right| M_{\infty}\right\rangle
\end{aligned}
$$

where $M^{(j)}\left(\mu_{Q}\right)$ is the mass of a heavy-light meson with spin $j$ containing a heavy quark with pole mass $\mu_{Q} ; h(v)$ is the heavy-quark field in the $H Q E T$ with fixed four-velocity $v ;\left|M_{\infty}\right\rangle$ is the heavy-meson state in the heavy-quark limit normalized as $\left\langle M_{\infty}|\bar{h}(v) h(v)| M_{\infty}\right\rangle=1$; $D_{\perp}^{\mu} \equiv D^{\mu}-(v \cdot D) v^{\mu}$, with $D^{\mu} \equiv \partial^{\mu}-i g A^{\mu}$ being the gauge-covariant derivative; $\sigma_{\mu \nu} \equiv$ $\frac{i}{2}\left[\gamma_{\mu}, \gamma_{\nu}\right] ; G^{\mu \nu} \equiv \frac{i}{g}\left[D^{\mu}, D^{\nu}\right]$ is the gluon field-strength tensor; $d_{j}$ is a spin coefficient, which takes the value 3 and -1 in case of pseudoscalar and vector mesons, respectively.

The parameter $\bar{\Lambda}$, which is also related to the trace anomaly of $Q C D$ [3] $(\bar{\Lambda}=$ $\left\langle M_{\infty}\left|\bar{h}(v) \frac{\beta\left(\alpha_{s}\right)}{4 \alpha_{s}} G_{\mu \nu} G^{\mu \nu} h(v)\right| M_{\infty}\right\rangle$, with $\beta$ and $\alpha_{s}$ being the Gell-Mann-Low function and the running strong coupling constant), represents the heavy-quark binding energy and measures the mass of the light degrees of freedom in the static colour source provided by the heavy quark. The quantity $-\lambda_{1} / 2 \mu_{Q}$ is often referred to as the heavy-quark kinetic energy, while $-d_{j} \lambda_{2} / 2 \mu_{Q}$ is the leading contribution of the chromomagnetic interaction to the heavy-meson mass. Thus, the $H Q E T$ expansion of the meson mass $M^{(j)}\left(\mu_{Q}\right)$ reads as [1]

$$
M^{(j)}\left(\mu_{Q}\right)=\mu_{Q}+\bar{\Lambda}-\frac{\lambda_{1}+d_{j} \lambda_{2}}{2 \mu_{Q}}+\frac{\rho_{1}+d_{j} \rho_{2}}{4 \mu_{Q}^{2}}+O\left(\frac{1}{\mu_{Q}^{3}}\right)
$$

where the parameters $\rho_{1}$ and $\rho_{2}$ govern the $1 / \mu_{Q}^{2}$ correction terms. All the HQET parameters appearing in Eq. (2) contribute to the non-perturbative power corrections (up to the order $\left.\left(\Lambda_{Q C D} / \mu_{Q}\right)^{3}\right)$ to the free-quark limit for the inclusive lepton spectrum in heavy-meson semileptonic decays.

Experimental information on the $H Q E T$ parameter $\lambda_{2}$ is directly available from meson spectroscopy through the $B^{*}-B$ mass splitting, yielding $\lambda_{2}=\left(M_{B *}^{2}-M_{B}^{2}\right) / 4+O\left(1 / \mu_{Q}\right) \simeq$ 
$0.12 \mathrm{GeV}^{2}$. As for $\lambda_{1}$ and $\bar{\Lambda}$, several attempts have recently been performed in order to extract them from model-independent analyses of the inclusive lepton spectrum of beauty and charm decays [4, 5, 6]. The most recent analysis of Ref. [4] has provided the values $\bar{\Lambda}=(0.33 \pm 0.11) \mathrm{GeV}$ and $-\lambda_{1}=(0.17 \pm 0.10) \mathrm{GeV}^{2}$, where the quoted uncertainties arise mainly from the non-perturbative $\left(\Lambda_{Q C D} / \mu_{b}\right)^{3}$ corrections in the inclusive $B$-meson lepton spectrum.

As far as the theoretical point of view is concerned, the $H Q E T$ hadronic parameters have recently been investigated within the lattice formulation of $H Q E T$ [7] and the $Q C D$ sum rule $(Q C D-S R)$ techniques [3, 8, 9] (for earlier $Q C D-S R$ analyses see Ref. [10]). The most interesting situation occurs for the $H Q E T$ parameter $\lambda_{1}$. In Ref. [8 the $Q C D-S R$ value $-\lambda_{1}=(0.52 \pm 0.12) \mathrm{GeV}^{2}$ has been obtained, consistently with the lower bound $-\lambda_{1}>3 \lambda_{2} \simeq 0.36 \mathrm{GeV}^{2}$ derived in Ref. [3] within the so-called $Q C D-S R$ in the small velocity limit. However, in Ref. [11] such a lower bound has been weakened significantly due to the effects of high-order radiative corrections. In Ref. [9] a re-analysis of the $Q C D$ sum rules, based on the virial theorem, has yielded a substantially smaller value, $-\lambda_{1}=(0.10 \pm$ $0.05) \mathrm{GeV}^{2}$. Moreover, the estimate $-\lambda_{1}=-(0.09 \pm 0.14) \mathrm{GeV}^{2}$ has been extracted from recent lattice $Q C D$ simulations [0]. It seems clear therefore that a small value of $-\lambda_{1}$ may be suggested theoretically and is consistent with available model-independent information. On the contrary, within the constituent quark $(C Q)$ model the existing predictions for $-\lambda_{1}$ lie in the range $0.4 \div 0.6 \mathrm{GeV}^{2}$ (cf. the recent works of Refs. [12, 13]). As a matter of fact, the quark model expectation is $-\lambda_{1}=\left\langle p^{2}\right\rangle \equiv\left\langle M_{\infty}\left|p^{2}\right| M_{\infty}\right\rangle$, where $\vec{p}$ is the heavy-quark threemomentum in the heavy meson and $p^{2}=|\vec{p}|^{2}$. Typically, one has $\left\langle p^{2}\right\rangle \simeq 0.4 \div 0.6 \mathrm{GeV}^{2}$, depending on the detailed shape of the heavy-quark momentum distribution inside the heavylight meson (cf. Refs. [12, 13]). However, we point out that the result $-\lambda_{1}=\left\langle p^{2}\right\rangle$ is characteristic of $C Q$ models in which a non-relativistic interquark potential, which is more appropriate in case of heavy-heavy mesons, is adopted for the description of heavy-light mesons, as it has been done in Refs. [12, 13] and [14]. The aim of this letter is indeed to show that in case of heavy-light mesons the $C Q$ model $(C Q M)$ prediction for $-\lambda_{1}$ is sharply sensitive to relativistic effects in the $C Q$ interaction, which may lead to substantial deviations from the non-relativistic expectation $-\lambda_{1}=\left\langle p^{2}\right\rangle$.

Let us consider a $Q \bar{q}$ meson, containing a heavy quark $Q$ with constituent mass $m_{Q}$ and a lighter partner (anti)quark $\bar{q}$ with constituent mass $m_{\bar{q}}$. Limiting ourselves to $S$-wave mesons for simplicity, the effective $C Q$ Hamiltonian can generally be written in the form (cf. 15)

$$
\left\{\sqrt{m_{Q}^{2}+p^{2}}+\sqrt{m_{\bar{q}}^{2}+p^{2}}+V_{Q \bar{q}}\right\} w_{Q \bar{q}}\left(p^{2}\right)|j \mu\rangle=M_{Q \bar{q}}^{(j)} w_{Q \bar{q}}\left(p^{2}\right)|j \mu\rangle
$$

where $M_{Q \bar{q}}^{(j)}$ is the meson mass, $V_{Q \bar{q}}$ the effective interquark potential, $w_{Q \bar{q}}\left(p^{2}\right)$ the $S$-wave radial function and $|j \mu\rangle$ the $C Q$ spin wave function

\footnotetext{
${ }^{b}$ Equation (3) is not manifestly covariant; however, following Ref. [16], one can apply a unitary transformation to the canonical wave function $w_{Q \bar{q}}\left(p^{2}\right)|j \mu\rangle$ in order to satisfy explicitly the requirements of Poincaré covariance. Of course, such a unitary transformation does not affect the eigenvalues of Eq. (3), which are the quantities of interest in this work.
} 
Assuming that the heavy-quark static limit of $V_{Q \bar{q}}$ is well defined, $V_{Q \bar{q}}^{\infty} \equiv \lim _{m_{Q} \rightarrow \infty} V_{Q \bar{q}}$, one can construct a heavy-quark expansion for the eigenvalue $M_{Q \bar{q}}^{(j)}$ of Eq. (3) which is similar to the HQET expansion (2), but expressed in terms of powers of the inverse heavy-quark constituent mass, namely

$$
M_{Q \bar{q}}^{j}\left(m_{Q}\right)=m_{Q}+\bar{\Lambda}^{(C Q M)}-\frac{\lambda_{1}^{(C Q M)}+d_{j} \lambda_{2}^{(C Q M)}}{2 m_{Q}}+\frac{\rho_{1}^{(C Q M)}+d_{j} \rho_{2}^{(C Q M)}}{4 m_{Q}^{2}}+O\left(\frac{1}{m_{Q}^{3}}\right)
$$

where

$$
\bar{\Lambda}^{(C Q M)}=\left\langle M_{\infty}\left|\sqrt{m_{\bar{q}}^{2}+p^{2}}+V_{Q \bar{q}}^{\infty}\right| M_{\infty}\right\rangle
$$

while $\lambda_{1}^{(C Q M)}$ and $\rho_{1}^{(C Q M)}$ are the relevant $C Q M$ parameters in the heavy-quark expansion of the spin-averaged binding energy $\bar{\Lambda}_{Q \bar{q}}$

$$
\bar{\Lambda}_{Q \bar{q}} \equiv \frac{M_{Q \bar{q}}^{(0)}+3 M_{Q \bar{q}}^{(1)}}{4}-m_{Q}=\bar{\Lambda}^{(C Q M)}-\frac{\lambda_{1}^{(C Q M)}}{2 m_{Q}}+\frac{\rho_{1}^{(C Q M)}}{4 m_{Q}^{2}}+O\left(1 / m_{Q}^{3}\right)
$$

and $\lambda_{2}^{(C Q M)}$ and $\rho_{2}^{(C Q M)}$ appear in the heavy-quark expansion of the meson mass splitting

$$
M_{Q \bar{q}}^{(1)}-M_{Q \bar{q}}^{(0)}=\frac{2 \lambda_{2}^{(C Q M)}}{m_{Q}}-\frac{\rho_{2}^{(C Q M)}}{m_{Q}^{2}}+O\left(1 / m_{Q}^{3}\right)
$$

If the spin-independent part of the interquark interaction $V_{Q \bar{q}}$ does not depend on the constituent mass $m_{Q}$, then one gets $-\lambda_{1}^{(C Q M)}=\left\langle p^{2}\right\rangle$, while the implicit $m_{Q^{-}}$dependence of the radial wave function $w_{Q \bar{q}}\left(p^{2}\right)$ leads to $\rho_{1}^{(C Q M)} \neq 0$.

In heavy $Q \bar{Q}$ quarkonia the non-relativistic approximation is expected to be quite reasonable and, therefore, for such systems a local potential of the Cornell type, containing an approximately linear-confining term plus the one-gluon-exchange contribution, can be adopted. As a matter of fact, the non-relativistic expansion of the interquark interaction has been found to be almost adequate for the description of charmonium and bottonium mass spectra (cf. Ref. [17] and see also Ref. [18] for a recent discussion of semi-relativistic interactions in heavy quarkonia). In Cornell-type potentials the spin-independent Coulomblike term, originating from the one-gluon-exchange interaction, dominates at small interquark distances and governs the high-momentum tail of the ground-state meson wave function, affecting therefore significantly the value of $\left\langle p^{2}\right\rangle$ (cf. Ref. [16]).

If a local Cornell-type interaction is adopted in heavy-light mesons, one typically gets $\left\langle p^{2}\right\rangle \simeq 0.4 \div 0.6 \mathrm{GeV}^{2}$ (cf. Refs. [12, 13]), depending mainly on the specific value adopted for the (effective) running coupling constant, which governs directly the strength of the Coulomb-like interaction term $\mathrm{l}$. However, the relativistic effects in the interquark

${ }^{c}$ In the $I S G W$ model [14] a local Cornell-type potential is adopted and the ground-state meson wave function is approximated by a gaussian ansätz, which yields $\left\langle p^{2}\right\rangle \simeq 0.28 G e V^{2}$. However, such an approximation underestimates significantly the value of $\left\langle p^{2}\right\rangle$ corresponding to the exact ground-state of the $I S G W$ Hamiltonian, because the effects of the Coulomb-like interaction on the high-momentum tail of the ground-state meson wave function are not properly taken into account by a soft gaussian ansätz (see Ref. 16]). 
potential are expected to be significantly larger in heavy-light mesons than in heavy-heavy ones, mainly because the momentum of the light quark should be of the order of $\Lambda_{Q C D}$ or even larger, which implies $p / m_{\bar{q}} \gtrsim 1$. As a consequence, the non-relativistic expansion of the interquark potential is inaccurate in heavy-light mesons and the non-relativistic limit can almost be reached only when both $m_{Q}$ and $m_{\bar{q}}$ are large enough (see Refs. [15, 16, 18]). The main qualitative features of the relativistic effects in the interquark interaction are the smearing of the $C Q$ coordinates and the momentum (energy) dependence of the strength of the interaction (see, e.g., Ref. [15]). The former feature can usually be accomplished via the introduction of a smearing function, which extends over interquark distances of the order of the inverse quark masses. The important point is that the relativistic effects make the various components of the effective interaction $V_{Q \bar{q}}$ dependent explicitly upon the masses of both interacting $C Q$ 's. In particular, the dependence of $V_{Q \bar{q}}$ upon $m_{Q}$, driven by the relativistic corrections, can lead to $1 / m_{Q}$ corrections to its heavy-quark static limit $V_{Q \bar{q}}^{\infty}=\lim _{m_{Q} \rightarrow \infty} V_{Q \bar{q}}$, yielding $-\lambda_{1}^{(C Q M)} \neq\left\langle p^{2}\right\rangle$, while, on the contrary, in case of the heavy-quark static interaction $V_{Q \bar{q}}^{\infty}$ one always gets $-\lambda_{1}^{(C Q M)}=\left\langle p^{2}\right\rangle$, as in the case of the non-relativistic approximation (i.e., when also $m_{\bar{q}} \rightarrow \infty$ ). The $1 / m_{Q}$ corrections to the heavy-quark static approximation $V_{Q \bar{q}}^{\infty}$ are expected to depend on the mass $m_{\bar{q}}$ of the partner (anti)quark and, therefore, the comparison of the meson masses predicted by $V_{Q \bar{q}}$ and its heavy-quark static limit $V_{Q \bar{q}}^{\infty}$ can provide an estimate of the relevance of the relativistic effects in the interquark interaction.

In Ref. 15 a phenomenological parametrization of the smearing function as well as of the energy dependence of the interquark interaction has been developed and applied to the calculation of the meson mass spectra. Though the potential model of Godfrey and Isgur $(G I)$ [15] is far from being fully justified in terms of $Q C D$ and, in particular, its relativized features should be considered only as a first step towards a full relativistic treatment of the interquark interaction, it is remarkable the fact that the GI potential allows the reproduction of a large number of meson energy levels from the pion to the upsilon. In what follows we will consider the relativized $G I$ interaction, $V_{G I}$, and its heavy-quark static limit, $V_{G I}^{\infty} \equiv \lim _{m_{Q} \rightarrow \infty} V_{G I}$.

The spin-averaged binding energy $\bar{\Lambda}_{Q \bar{q}}$ has been calculated by solving Eq. (3) for the ground-state of pseudoscalar and vector heavy-light mesons at various values of the heavy-quark mass $m_{Q}$. The results obtained at $m_{\bar{q}}=0.220,0.419$ and also $1.628 \mathrm{GeV}$, which correspond to the $u$ - $(d-), s$ - and $c$-quark constituent masses adopted in Ref. [15], are reported in Fig. 1 as a function of the inverse heavy-quark mass, $1 / m_{Q}$. A quadratic fit in $1 / m_{Q}$ (see Eq. (可)) has been applied to the calculated values of $\bar{\Lambda}_{Q \bar{q}}$ for $m_{Q} \geq 5 \mathrm{GeV}$ and the results are shown in Fig. 1 by the dashed and solid lines. Let us notice that the same quadratic fits reproduce also the calculated values of $\bar{\Lambda}_{Q \bar{q}}$ up to $m_{Q} \simeq 2 \mathrm{GeV}$ within few \%. The values obtained for $\bar{\Lambda}^{(C Q M)}, \lambda_{1}^{(C Q M)}$ and $\rho_{1}^{(C Q M)}$ are reported in Table 1. It can clearly be seen that the values of both $\lambda_{1}^{(C Q M)}$ and $\rho_{1}^{(C Q M)}$, corresponding to the relativized $G I$ interaction and its static limit $G I^{\infty}$, differ remarkably even when $m_{\bar{q}}$ is around the $c$-quark mass $d$. Though the precise value of $\lambda_{1}^{(C Q M)}$ and $\rho_{1}^{(C Q M)}$ depends

\footnotetext{
${ }^{d}$ The values of $\bar{\Lambda}^{(C Q M)}$ for the $G I$ and $G I^{\infty}$ potentials clearly coincide, because the $G I^{\infty}$ interaction is the heavy-quark static limit of the GI one (see Eq. (5)).
} 
strongly on the way the relativistic effects are parametrized in the $C Q$ potential, the results presented in Fig. 1 and Table 1 clearly show that the $C Q M$ parameters $\lambda_{1}^{(C Q M)}$ and $\rho_{1}^{(C Q M)}$ exhibit a large sensitivity to the relativistic effects in the interquark interaction, which may lead to substantial deviations from the heavy-quark static expectations. In particular, the relativistic effects of the $G I$ potential model make $-\lambda_{1}^{(C Q M)}$ substantially smaller than the heavy-quark static expectation. Note also that: i) the absolute value of $\rho_{1}^{(C Q M)}$ increases as the partner (anti)quark mass $m_{\bar{q}}$ increases; ii) the relativistic effects parametrized in the $G I$ interaction reduce remarkably the absolute value of $\rho_{1}^{(C Q M)}$, which, we remind, contributes to the non-perturbative $\left(\Lambda_{Q C D} / m_{Q}\right)^{3}$ corrections in the inclusive lepton spectrum for heavymeson semileptonic decays. Neverthless, the $G I$ prediction for $\rho_{1}^{(C Q M)}$ at $m_{\bar{q}}=0.220 \mathrm{GeV}$ (see Table 1$)$ is about twice the value of $\rho_{1}\left(\simeq 0.03 \mathrm{GeV}^{3}\right)$ quoted in Ref. [四] as the result of the vacuum-saturation approximation.

In case of the GI potential the values of $\lambda_{2}^{(C Q M)}$ and $\rho_{2}^{(C Q M)}$, obtained from the quadratic fit of the calculated meson mass splitting (7) for $m_{Q} \geq 5 \mathrm{GeV}$, are: $\lambda_{2}^{(C Q M)}=$ 0.149, 0.145, 0.215 GeV and $\rho_{2}^{(C Q M)}=0.0026,0.0423,0.453 \mathrm{GeV}^{3}$ at $m_{\bar{q}}=0.220,0.419$, $1.628 \mathrm{GeV}$, respectively. Note that the value of $\lambda_{2}^{(C Q M)}$ depends only slightly upon the partner (anti)quark mass $m_{\bar{q}}$, while $\rho_{2}^{(C Q M)}$ turns out to be quite small at $m_{\bar{q}}=0.220$ and $0.419 \mathrm{GeV}$, but significantly large for $m_{\bar{q}}$ around the $c$-quark mass. For the $G I^{\infty}$ interaction one clearly has $\lambda_{2}^{(C Q M)}=\rho_{2}^{(C Q M)}=0$, because all the hyperfine terms are vanishing in the heavy-quark static limit.

The values of the $C Q M$ parameters $\bar{\Lambda}^{(C Q M)}, \lambda_{1}^{(C Q M)}$ and $\lambda_{2}^{(C Q M)}$, calculated at $m_{\bar{q}}=$ $0.220 \mathrm{GeV}$ using the $G I$ potential, are directly compared with the HQET hadronic parameters $\bar{\Lambda}, \lambda_{1}$ and $\lambda_{2}$ obtained within various approaches. It can be seen that the $G I$ value for $\bar{\Lambda}^{(C Q M)}$ is in agreement with the result of Ref. 四, while the GI value of $\lambda_{1}^{(C Q M)}$ strongly differs (even in sign) with the findings of Ref. [4], but it is consistent with the central value of the lattice $Q C D$ simulations of Ref. [7]. Moreover, the $G I$ value for $\lambda_{2}^{(C Q M)}$ is quite close to the experimental one, because the $G I$ interaction is tailored to reproduce the hyperfine meson mass splittings. However, the direct comparison between the HQET and $C Q M$ hadronic parameters is meaningful only if the $C Q$ mass $m_{Q}$ is assumed to be equal to the pole heavy-quark mass $\mu_{Q}$ (see the expansions (2) and (4)). Since in the $C Q$ model the effects of the frozen degrees of freedom are expected to be parametrized also via the $C Q$ masses, one can speculate that $m_{Q} \neq \mu_{Q}$ and consider a heavy-quark expansion of the type

$$
m_{Q}=\mu_{Q}+\bar{\Lambda}^{Q}-\frac{\lambda^{Q}}{2 \mu_{Q}}+\frac{\rho^{Q}}{4 \mu_{Q}^{2}}+O\left(1 / \mu_{Q}^{3}\right)
$$

which may lead to

$$
\begin{aligned}
\bar{\Lambda} & =\bar{\Lambda}^{(C Q M)}+\bar{\Lambda}^{Q} \\
\lambda_{1} & =\lambda_{1}^{(C Q M)}+\lambda^{Q} \\
\rho_{1} & =\rho_{1}^{(C Q M)}+\rho^{Q}+2 \bar{\Lambda}^{Q} \lambda_{1}^{(C Q M)} \\
\lambda_{2} & =\lambda_{2}^{(C Q M)} \\
\rho_{2} & =\rho_{2}^{(C Q M)}+2 \bar{\Lambda}^{Q} \lambda_{2}^{(C Q M)}
\end{aligned}
$$


In the heavy-quark limit one could simply expect that any difference between constituent and current quarks disappear, which means $\lim _{\mu_{Q} \rightarrow \infty}\left(m_{Q}-\mu_{Q}\right)=0$, i.e. $\bar{\Lambda}^{Q}=0$. This implies $\bar{\Lambda}=\bar{\Lambda}^{(C Q M)}$ (as it is suggested by the comparison with the result of Ref. [4]) and $\rho_{2}=\rho_{2}^{(C Q M)}$, while one still has $\lambda_{1}=\lambda_{1}^{(C Q M)}+\lambda^{Q}$ and $\rho_{1}=\rho_{1}^{(C Q M)}+\rho^{Q}$ with unknown values for $\lambda^{Q}$ and $\rho^{Q}$ ll. In order to get rid of any possible relation between $m_{Q}$ and $\mu_{Q}$, one can consider the difference between the parameter $\lambda_{1}$ corresponding to different flavours of the partner (anti)quark; indeed, from Eq. (9) one easily gets

$$
\lambda_{1}\left(\bar{q}^{\prime}\right)-\lambda_{1}(\bar{q})=\lambda_{1}\left(\bar{q}^{\prime}\right)^{(C Q M)}-\lambda_{1}(\bar{q})^{(C Q M)}
$$

The results obtained for Eq. (10) using the $G I$ and $G I^{\infty}$ potentials are reported in Table 3. It can be seen that both the $G I$ and $G I^{\infty}$ predictions for the quantity $\lambda_{1}(u)-\lambda_{1}(s)$ are consistent with the recent lattice result of Ref. [7], while the difference $\lambda_{1}(u)-\lambda_{1}(c)$ (i.e., the difference of the hadronic parameter $\lambda_{1}$ in the $B_{u(d)}$ and $B_{c}$ mesons) is sharply sensitive to the $1 / m_{Q}$ corrections to the heavy-quark static limit and, therefore, its determination could provide information on the relativistic effects in the interquark interaction.

In conclusion, the spin-averaged binding energy and the hyperfine mass splitting of heavy-light mesons have been investigated within the constituent quark model as a function the inverse heavy-quark mass. It has been shown that the so-called heavy-quark kinetic energy, $-\lambda_{1}^{(C Q M)} / 2 m_{Q}$, may differ remarkably from the non-relativistic expectation $\left\langle p^{2}\right\rangle / 2 m_{Q}$, thanks to relativistic effects in the effective interquark potential, which may produce substantial $1 / m_{Q}$ corrections to the heavy-quark static limit. These corrections, which depend on the mass $m_{\bar{q}}$ of the partner (anti)quark in the heavy meson, have been found to be significant also for $m_{\bar{q}}$ around the $c$-quark mass. Moreover, the $1 / m_{Q}^{2}$ terms in the heavy-quark expansion of the meson mass are predicted to be quite small for $m_{\bar{q}}$ around the $u$ - and $s$ quark constituent masses, but significantly larger for $m_{\bar{q}}$ around the $c$-quark mass. Finally, it has been suggested that the determination of the difference of the hadronic parameter $\lambda_{1}$ in the $B_{u(d)}$ and $B_{c}$ mesons can provide information about the strength of the relativistic effects in the interquark interaction.

Acknowledgments. The author thanks Fabio Cardarelli for helpful discussions and a careful reading of the manuscript.

\section{References}

[1] For a review see, e.g., M. Neubert: Phys. Rep. 245 (1994) 259, and references therein quoted.

[2] N. Isgur and M.B. Wise: Phys. Lett. B232 (1989) 113; ib. B237 (1990) 527; Nucl. Phys. B348 (1991) 276.

\footnotetext{
${ }^{e}$ Using $\lambda_{1}=-0.17 \pm 0.10 \mathrm{GeV}^{2}$ 䐗 and the $G I$ value $\lambda_{1}^{(C Q M)}=0.089 \mathrm{GeV}^{2}$, one gets $\lambda^{Q}=-0.26 \pm$ $0.10 \mathrm{GeV}^{2}$, which would be interpreted as the average value of the current heavy-quark momentum squared in the constituent heavy-quark.
} 
[3] I. Bigi, M. Shifman, N.G. Uraltsev and A. Vainshtein: Phys. Rev. D52 (1995) 196; Int. J. Mod. Phys. A9 (1994) 2467. I. Bigi, A.G. Grozin, M. Shifman, N.G. Uraltsev and A. Vainshtein: Phys. Lett. B339 (1994) 160.

[4] M. Gremm, A. Kapustin, Z. Ligeti and M.B. Wise: Phys. Rev. Lett. 77 (1996) 20. M. Gremm and I. Stewart: Phys. Rev. D55 (1997) 1226.

[5] A.F. Falk, M. Luke and M.J. Savage: Phys. Rev. D53 (1996) 6316.

[6] V. Chernyak: Nucl. Phys. B457 (1995) 96.

[7] V. Giménez, G. Martinelli and C.T. Sachrajda: Nucl. Phys. B486 (1997) 227; Phys. Lett. B393 (1997) 124.

[8] P. Ball and V.M. Braun: Phys. Rev. D49 (1994) 2472. E. Bagan, P. Ball, V. Braun and V. Gosdzinsky: Phys. Lett. B342 (1995) 362.

[9] M. Neubert: Phys. Lett. B389 (1996) 727.

[10] M. Neubert: Phys. Rev. D46 (1992) 1076. V. Eletsky and E. Shuryak: Phys. Lett. B276 (1992) 191. E. Bagan, P. Ball, V. Braun and H. Dosch: Phys. Lett. B278 (1992) 457.

[11] A. Kapustin, Z. Ligeti, M.B. Wise and B. Grinstein: Phys. Lett. B375 (1996) 327. H. Davoudiasl and A.K. Lebovich: Phys. Lett. B403 (1997) 323.

[12] F. De Fazio: Mod. Phys. Lett. A11 (1996) 2693.

[13] D. S. Hwang, C.S. Kim and W. Namgung: Phys. Rev. D54 (1996) 5620; Phys. Lett. B406 (1997) 117.

[14] D. Scora and N. Isgur: Phys. Rev. D52 (1995) 2783.

[15] S. Godfrey and N. Isgur: Phys. Rev. D32 (1985) 189.

[16] S. Simula: Phys. Lett. B373 (1996) 193. See also F. Cardarelli et al.: Phys. Lett. B332 (1994) 1; Phys. Lett. B349 (1995) 393; Phys. Rev. D53 (1996) 6682 and I.L. Grach et al.: Phys. Lett. B385 (1996) 317.

[17] For a review see, e.g., W. Lucha, F.F. Schöberl and D. Gromes: Phys. Rep. 200 (1991) 127 and references therein quoted.

[18] N. Brambilla and A. Vairo: Phys. Rev. D55 (1997) 3794; Phys. Lett. B407 (1997) 167. 

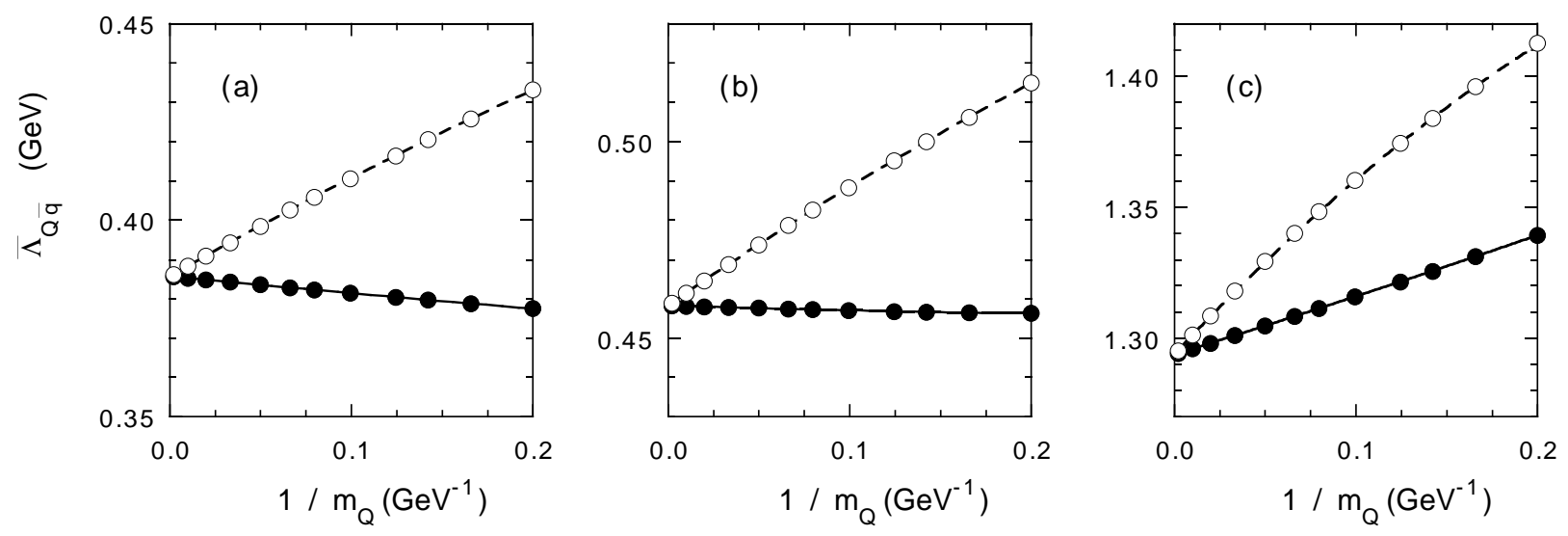

Figure 1. Values of the spin-averaged binding energy $\bar{\Lambda}_{Q \bar{q}}$ (Eq. (6)) as a function of the inverse heavy-quark constituent mass $1 / m_{Q}$, obtained from the ground-state eigenvalue of Eq. (3) calculated in case of pseudoscalar and vector heavy-light mesons adopting the GI potential model [15] (full dots) and its heavy-quark static limit $G I^{\infty}$ (open dots). In (a, b, c) the mass of the partner (anti)quark is $m_{\bar{q}}=0.220,0.419$ and $1.628 \mathrm{GeV}$, respectively. The dashed and solid lines are quadratic fits in $1 / m_{Q}$ of the calculated values of $\bar{\Lambda}_{Q \bar{q}}$ for $m_{Q} \geq 5 \mathrm{GeV}$.

Table 1. Values of the $C Q M$ parameters $\bar{\Lambda}^{(C Q M)}, \lambda_{1}^{(C Q M)}$ and $\rho_{1}^{(C Q M)}$, obtained from the quadratic fit of the calculated values of the spin-averaged binding energy $\bar{\Lambda}_{Q \bar{q}}$ (6) (see text and Fig. 1). The values of the partner (anti)quark mass $m_{\bar{q}}$ are given in $G e V$ and correspond to the $u$ - $(d-), s$ - and $c$-quark constituent masses of Ref. [15.

\begin{tabular}{||c|c||c|c|c||}
\hline Pot. & $C Q M$ param. & $m_{\bar{q}}=0.220$ & $m_{\bar{q}}=0.419$ & $m_{\bar{q}}=1.628$ \\
\hline \hline \multirow{3}{*}{$G I$} & $\Lambda^{(C Q M)}(G e V)$ & 0.386 & 0.458 & 1.294 \\
\cline { 2 - 5 } & $-\lambda_{1}^{(C Q M)}\left(\mathrm{GeV}^{2}\right)$ & -0.089 & -0.031 & 0.426 \\
\cline { 2 - 5 } & $\rho_{1}^{(C Q M)}\left(\mathrm{GeV}^{3}\right)$ & 0.063 & 0.103 & 0.346 \\
\hline \hline \multirow{3}{*}{$G I^{\infty}$} & $\bar{\Lambda}^{(C Q M)}\left(\mathrm{GeV}^{(C Q}\right)$ & 0.386 & 0.458 & 1.294 \\
\cline { 2 - 5 } & $-\lambda_{1}^{(C Q M)}\left(\mathrm{GeV}^{2}\right)$ & 0.523 & 0.635 & 1.474 \\
\cline { 2 - 5 } & $\rho_{1}^{(C Q M)}\left(\mathrm{GeV}^{3}\right)$ & -0.514 & -0.717 & -2.966 \\
\hline \hline
\end{tabular}


Table 2. Comparison of the values of the HQET hadronic parameters $\bar{\Lambda}, \lambda_{1}$ and $\lambda_{2}$ (Eq. (1)) obtained within various approaches, with the $C Q M$ parameters $\bar{\Lambda}^{(C Q M)}, \lambda_{1}^{(C Q M)}$ and $\lambda_{2}^{(C Q M)}$ (Eqs. (5-7)), calculated in the present work at $m_{\bar{q}}=0.220 \mathrm{GeV}$ using the GI interaction [15].

\begin{tabular}{|c|c|c|c|c|}
\hline$\overline{R e f}$. & Method & $\bar{\Lambda}(\mathrm{GeV})$ & $-\lambda_{1}\left(G e V^{2}\right)$ & $\lambda_{2}\left(G e V^{2}\right)$ \\
\hline 3 & $Q C D-S R$ & $>0.5$ & $>0.36$ & -- \\
\hline 8 & $Q C D-S R$ & $0.4 \div 0.5$ & $0.52 \pm 0.12$ & $0.11 \pm 0.04$ \\
\hline 9] & $Q C D-S R$ & -- & $0.10 \pm 0.05$ & $0.15 \pm 0.03$ \\
\hline |7] & lattice & $0.18 \pm 0.03$ & $-0.09 \pm 0.14$ & $0.07 \pm 0.01$ \\
\hline (41) & exp. & $0.33 \pm 0.11$ & $0.17 \pm 0.10$ & $\simeq 0.12$ \\
\hline [5] & exp. & 0.45 & 0.10 & $\simeq 0.12$ \\
\hline (6) & exp. & -- & 0.14 & $\simeq 0.12$ \\
\hline $\mid 12$ & $C Q M$ & 0.35 & 0.66 & -- \\
\hline 13 & $C Q M$ & $0.5 \div 0.6$ & $0.4 \div 0.6$ & -- \\
\hline this work & rel. $C Q M$ & 0.386 & -0.089 & 0.149 \\
\hline
\end{tabular}

Table 3. Values of the difference of the HQET parameters $\lambda_{1}$ (in $\mathrm{GeV}^{2}$ ) corresponding to different flavours of the partner (anti)quark predicted by the $G I$ [15] and $G I^{\infty}$ potentials through Eq. (10). The result of the lattice QCD simulation of Ref. [0] is also reported.

\begin{tabular}{||c||c|c||}
\hline Potential & $\lambda_{1}(u)-\lambda_{1}(s)$ & $\lambda_{1}(u)-\lambda_{1}(c)$ \\
\hline \hline$G I$ & 0.058 & 0.515 \\
\hline$G I^{\infty}$ & 0.112 & 0.951 \\
\hline \hline lattice [7] & $0.09 \pm 0.04$ & -- \\
\hline \hline
\end{tabular}

